\title{
Registro de Culicidae de importância epidemiológica na área rural de Manaus, Amazonas
}

\author{
Record of epidemiologically important Culicidae in the \\ rural area of Manaus, Amazonas
}

\author{
Maria das Graças Vale Barbosa ${ }^{1,2,3}$, Nelson Ferreira Fé1, \\ Alexandre Herculano Ribera Marcião ${ }^{4}$, Ana Paula Thomé da Silva ${ }^{4}$, \\ Wuelton Marcelo Monteiro ${ }^{1,2}$, Marcus Vinitius de Farias Guerra ${ }^{1,4}$ \\ e Jorge Augusto de Oliveira Guerra ${ }^{1,2}$
}

\begin{abstract}
RESUMO
A biodiversidade da Região Amazônica inclui diversas espécies de vetores artrópodes em seus diferentes ecótopos, possibilitando o surgimento de doenças como malária, filarioses e arboviroses. De agosto de 2001 a julho de 2002, realizou-se coletas de culicídeos no domicílio, peridomicílio e nas matas da Comunidade São João, área rural de Manaus, Amazonas. Foram capturados 1.240 culicídeos, pertencentes às subfamílias Culicinae (99\%) e Anophelinae (1\%), somando 50 espécies. 0 predomínio entre as tribos foi nitidamente de Culicini, com 904 (72,9\%) exemplares, destacando-se as espécies Culex usquatus (22,6\%) e Culex quinquefasciatus (17,7\%). Do total de culicídeos, 1.077 (86,9\%) exemplares foram capturados no interior das matas, 101 $(8,1 \%)$ no peridomicílio e 62 (5\%) no intradomicílio. 0 ecótopo com maior diversidade de espécies foi a mata. Assinalou-se a presença de Anopheles darlingi, Anopheles triannulatus, Aedes aegypti, Haemagogus janthinomys e outros vetores comprovados ou potenciais.
\end{abstract}

Palavras-chaves: Culicidae. Diversidade de espécies. Malária. Filarioses. Arboviroses.

\begin{abstract}
The biodiversity of the Amazon region includes many species of arthropod vectors in different ecotopes, thus enabling occurrences of diseases like malaria, filariasis and arbovirosis. From August 2001 to July 2002, we gathered culicids from inside homes, from areas surrounding these homes and from forested areas of the São João Community, in the rural zone of Manaus, State of Amazonas. 1240 specimens were collected, belonging to the Culicinae (99\%) and Anophelinae (1\%) subfamilies, with 50 species. The Culicini tribe clearly predominated, with 904 specimens (72.9\%), and the species Culex usquatus (22.6\%) and Culex quinquefasciatus (17.7\%) were prominent. Out of the total number of culicids, 1,077 (86.9\%) were caught in the forests, $101(8.1 \%)$ in the areas surrounding homes and $62(5 \%)$ inside homes. Forests were the ecotope that presented the highest species diversity. The presence of Anopheles darlingi, Anopheles triannulatus, Aedes aegypti, Haemagogus janthinomys and other proven or potential vectors was recorded.
\end{abstract}

Key-words: Culicidae. Species diversity. Malaria. Filariasis. Arbovirosis.

A área de abrangência da Floresta Amazônica apresenta enorme potencial de biodiversidade, incluindo diversas espécies de vetores artrópodes em seus diferentes ecótopos. Este fato propicia o surgimento de novas doenças, como as arboviroses, novos focos de endemias em locais onde não ocorriam e o ressurgimento de outros focos com surtos epidêmicos a partir das alterações ambientais provocadas pelo homem ${ }^{4} 31$. Nos ambientes desmatados, onde se instalam os trabalhadores, criamse condições propícias ao desenvolvimento de culicídeos vetores de agentes patogênicos ao homem ${ }^{20}$.
São freqüentes as investigações sobre a distribuição geográfica e sobre diversos aspectos do comportamento das espécies que compõem a fauna de culicídeos em áreas rurais ${ }^{11}{ }^{115}$. Sobre a diversidade destes insetos com destaque para os envolvidos na transmissão de agentes patogênicos, em localidades rurais da Região Amazônica estão os trabalhos de Natal e cols ${ }^{11}$, que evidenciaram predomínio do gênero Anopheles, com espécimes infectados por Plasmodium vivax e Plasmodium falciparum, e os de Fé e cols ${ }^{6}$, que registraram a presença de Haemagogus janthinomys, Haemagogus leucocelaenus, Sabethes belisarioi e

\footnotetext{
1. Gerencia de Entomologia da Fundação de Medicina Tropical do Amazonas, Manaus, AM. 2. Escola Superior de Ciências da Saúde, Universidade do Estado do Amazonas, Manaus, AM. 3. Centro Universitário Nilton Lins, Manaus, AM. 4. Faculdade de Medicina, Universidade Federal do Amazonas, Manaus, AM.

Endereço para correspondência: Dra. Maria das Graças Vale Barbosa. Gerência de Entomologia/FMTAM. Av. Pedro Teixeira 25, Dom Pedro, 69040-000 Manaus, AM.

Fax: $55923656-8269$

e-mail: gbarbosa@fmt.am.gov.br

Recebido em 05/05/2008

Aceito em 19/09/2008
} 
Aedes fulvus, espécies vetoras do vírus da febre amarela silvestre. $\mathrm{Na}$ Amazônia, verifica-se maior preocupação dos autores no estudo dos anofelinos, dada a importância que representam na transmissão da malária ${ }^{425} 26303132$.

O município de Manaus tem sofrido consideráveis alterações ambientais causadas pelo crescimento desordenado da sua área urbana e pela implementação de projetos agrícolas desorganizados na sua zona rural, aumentando o número de registros de diferentes enfermidades transmitidas por vetores ${ }^{24}$. A presente pesquisa tem como objetivo fazer uma descrição preliminar da fauna e do comportamento de culicídeos coletados em ambientes domiciliares, peridomiciliares e florestais na Comunidade Rural São João, núcleo rural de implantação recente do município de Manaus, Estado do Amazonas, contribuindo assim com dados que possam servir de base para ações relacionadas à saúde pública.

\section{MATERIAL E MÉTODOS}

Área de estudo. 0 trabalho foi realizado na Comunidade de São João, no km 4 da BR-174, que liga Manaus a Boa Vista (RR), no município de Manaus (0308'07’S, 6001'34”0). Nessa área vivem cerca de 500 famílias, distribuídas em uma área aproximada de $500 \mathrm{~m}$ de largura por $1.200 \mathrm{~m}$ de comprimento. Limita-se a oeste com a rodovia BR-174; à leste e ao sul, com áreas de floresta primária, semifragmentada, devido às atividades de extração madeireira; e ao norte, com uma área sem cobertura vegetal, onde são desenvolvidas atividades de avicultura e piscicultura. As matas vizinhas da comunidade são freqüentadas por muitos moradores para o desenvolvimento de atividades de extrativismo. Seu clima é tropical chuvoso e úmido, com temperatura média anual de $26^{\circ} \mathrm{C}$.

Coleta e identificação dos culicídeos. Durante doze meses consecutivos, de agosto 2001 a julho 2002, foram realizadas coletas passivas e ativas no intradomicílio, peridomicílio e nas matas. Em cada mês, durante cinco dias consecutivos, as coletas passivas foram realizadas, empregando-se armadilhas CDC (colocadas a um metro acima do solo) e armadilhas Disney; duas vezes na semana, em dias alternados, as coletas ativas foram feitas no horário das 18:00 às 21:00 horas, usando-se rede entomológica e tubos de vidro. Foram realizadas ainda varreduras em troncos de árvores do segundo ao quarto dia durante três horas diárias (8:00 às 11:00), em diversos pontos dentro da mata.

As coletas passivas (mata e peridomicílio), utilizando armadilhas do tipo CDC, eram iniciadas às 17:00 do primeiro dia (trocando-se as bolsas as 08:00 horas do dia seguinte) e encerradas as 08:00 horas do quinto dia. As armadilhas Disney foram colocadas a partir do primeiro dia, trocando-se as bandejas untadas com óleo mineral diariamente às 08:00 horas e retirandoas no quinto dia. Foram realizadas, ainda, inspeções intra e peridomiciliares, para a identificação de possíveis criadouros de culicídeos, registrando-se a presença de formas imaturas no interior de recipientes naturais ou artificiais.
A identificação dos culicídeos foi feita no Laboratório de Entomologia da Fundação de Medicina Tropical do Amazonas, onde os espécimes estão depositados. A identificação especifica foi realizada pelo segundo autor utilizando chaves dicotômicas $^{23578910}$.

\section{RESULTADOS}

Foram capturados 1.240 exemplares, pertencentes às subfamílias Culicinae (1.228 exemplares; 99\%) e Anophelinae (12; 1\%). Desta subfamília, foram coletados 4 exemplares de Anopheles darlingi e 8 de Anopheles triannulatus (Tabela 1). Da subfamília Culicinae foram registrados exemplares de 7 tribos, 13 gêneros e 48 espécies. 0 predomínio entre estas tribos foi nitidamente de Culicini, com 904 (72,9\%) exemplares, destacando-se as espécies Culex usquatus (280; 22,6\%), Culex quinquefasciatus (220;17,7\%), Culex declarator (189; $15,2 \%)$, Culex eastor (52; 4,2\%) e Culex innovator (46; 3,7\%) (Tabela 1). Foram identificados $213(17,2 \%)$ culicídeos da tribo Uranotaeniini, a segunda com maior número de exemplares. Desta, Uranotaenia ditaenionota foi a espécie mais freqüente (196; 15,8\%). Da subtribo Aedini, foram coletados 57 (4,6\%) exemplares, com destaque para Psorophora amazonica (43; 3,5\%). Foram coletados $31(2,5 \%)$ exemplares da tribo Mansoniini, 15 (1,2\%) de Sabethini, 3 (0,2\%) de Aedeomyiini e $3(0,2 \%)$ de Orthopodomyiini.

Do total de culicídeos, 1.077 (86,9\%) exemplares foram capturados no interior das matas, $101(8,1 \%)$ no peridomicílio e $62(5 \%)$ no intradomicílio. 0 ecótopo com maior diversidade de espécies foi a mata, com o registro de 39 espécies destacando-se Culex usquatus (280 exemplares), Uranotaenia uranotaenionota (196), Culex declarator (168), Culex quinquefasciatus (129) e Culex eastor (52). Registraram-se ainda neste ambiente a presença de Haemagogus janthinomys, Sabethes cyaneus e Psorophora ferox. No ambiente peridomiciliar foram registradas 17 espécies, com destaque para Culex quinquefasciatus (52 exemplares) e Culex declarator (11). No intradomicílio, foram registradas as espécies Culex quinquefasciatus (39 exemplares), Culex declarator (10), Culex nigripalpus (6), Psorophora amazonica (3), Aedes aegypti (2) e Mansonia pseudotitillans (2). Durante inspeções intra e peridomiciliares foi registrada a presença de larvas e pupas de Aedes aegypti no interior de um barril em um banheiro. Observaram-se 30 espécies exclusivas do ambiente florestal, 11 foram coletadas apenas no ambiente domiciliar e 11 nos dois ambientes.

A distribuição temporal das duas espécies vetoras mais freqüentes revelou que Culex quinquefasciatus (120 fêmeas e 70 machos), ocorreu durante todos os meses do ano, enquanto Culex declarator (173 fêmeas e 16 machos) ocorreu em seis meses (Figura 1). A primeira espécie foi mais abundante nos meses de janeiro e março e a segunda teve maior registro em março. 
Tabela 1 - Espécies de culicídeos coletadas na Comunidade Rural São João, Manaus, Amazonas, de agosto de 2001 a julbo de 2002.

\begin{tabular}{|c|c|c|c|c|c|}
\hline \multirow[b]{2}{*}{ Espécies } & \multirow[b]{2}{*}{ Peridomicílio } & \multirow[b]{2}{*}{ Intradomicílio } & \multirow[b]{2}{*}{ Mata } & \multicolumn{2}{|c|}{ Total } \\
\hline & & & & $\mathrm{n}^{\mathrm{o}}$ & $\%$ \\
\hline Aedeomyia (Aedeomyia) squamipennis & 3 & 0 & 0 & 3 & 0,2 \\
\hline Aedes (Ochlerotatus) serratus & 0 & 0 & 3 & 3 & 0,2 \\
\hline Aedes (Protomacleaya) argyrothorax & 0 & 0 & 2 & 2 & 0,2 \\
\hline Aedes (Stegomyia) aegypti & 0 & 2 & 0 & 2 & 0,2 \\
\hline Anopheles (Nyssorbynchus) darlingi & 4 & 0 & 0 & 4 & 0,3 \\
\hline Anopheles (Nyssorhynchus) triannulatus & 1 & 0 & 7 & 8 & 0,6 \\
\hline Coquilletidia (Rhynchotaenia) lynchi & 2 & 0 & 4 & 6 & 0,5 \\
\hline Coquilletidia (Rhynchotaenia) venezuelensis & 0 & 0 & 1 & 1 & 0,1 \\
\hline Coquilletidia (Rhynchotaenia) juxtamansonia & 0 & 0 & 1 & 1 & 0,1 \\
\hline Culex (Aedinus) accelerans & 0 & 0 & 1 & 1 & 0,1 \\
\hline Culex (Anoedioporpa) originator & 0 & 0 & 2 & 2 & 0,2 \\
\hline Culex (Carrolia) urichii & 0 & 0 & 1 & 1 & 0,1 \\
\hline Culex (Culex) declarator & 11 & 10 & 168 & 189 & 15,2 \\
\hline Culex (Culex) erythrotorax & 1 & 0 & 0 & 1 & 0,1 \\
\hline Culex (Culex) mauesensis & 0 & 0 & 3 & 3 & 0,2 \\
\hline Culex (Culex) nigripalpus & 0 & 6 & 0 & 8 & 0,6 \\
\hline Culex (Culex) quinquefasciatus & 52 & 39 & 129 & 220 & 17,7 \\
\hline Culex (Culex) usquatus & 0 & 0 & 280 & 280 & 22,6 \\
\hline Culex (Melanoconion) adamesi & 0 & 0 & 1 & 1 & 0,1 \\
\hline Culex (Melanoconion) atratus & 2 & 0 & 0 & 2 & 0,2 \\
\hline Culex (Melanoconion) dunni & 0 & 0 & 28 & 28 & 2,3 \\
\hline Culex (Melanoconion) eastor & 0 & 0 & 52 & 52 & 4,2 \\
\hline Culex (Melanoconion) innovator & 0 & 0 & 46 & 46 & 3,7 \\
\hline Culex (Melanoconion) pedro $i$ & 0 & 0 & 3 & 3 & 0,2 \\
\hline Culex (Melanoconion) pilosus & 5 & 0 & 0 & 5 & 0,4 \\
\hline Culex (Melanoconion) serratimarge & 0 & 0 & 1 & 1 & 0,1 \\
\hline Culex (Melanoconion) spissipes & 5 & 0 & 5 & 10 & 0,8 \\
\hline Culex (Melanoconion) theobaldi & 0 & 0 & 48 & 48 & 3,9 \\
\hline Culex (Melanoconion) ybarmis & 3 & 0 & 0 & 3 & 0,2 \\
\hline Haemagogus (Haemagogus) janthinomys & 0 & 0 & 13 & 13 & 1,0 \\
\hline Limatus durhami & 1 & 0 & 0 & 1 & 0,1 \\
\hline Limatus paraensis & 0 & 0 & 5 & 5 & 0,4 \\
\hline Mansonia (Mansonia) humeralis & 4 & 0 & 0 & 4 & 0,3 \\
\hline Mansonia (Mansonia) pseudotitillans & 0 & 2 & 2 & 4 & 0,3 \\
\hline Mansonia (Mansonia) titillans & 2 & 0 & 0 & 2 & 0,2 \\
\hline Orthopodomyia fascipes & 0 & 0 & 3 & 3 & 0,2 \\
\hline Psorophora (Grabhamia) cingulata & 2 & 0 & 1 & 3 & 0,2 \\
\hline Psorophora (Janthinosoma) amazonica & 0 & 3 & 43 & 46 & 3,7 \\
\hline Psorophora (Janthinosoma) ferox & 0 & 0 & 1 & 1 & 0,1 \\
\hline Psorophora (Janthinosoma) lutzii & 0 & 0 & 2 & 2 & 0,2 \\
\hline Sabethes (Sabethes) cyaneus & 0 & 0 & 1 & 1 & 0,1 \\
\hline Trichoprosopon digitatum & 0 & 0 & 1 & 1 & 0,1 \\
\hline Uranotaenia (Uranotaenia) calosomata & 0 & 0 & 5 & 5 & 0,4 \\
\hline Uranotaenia (Uranotaenia) ditaenionota & 0 & 0 & 196 & 196 & 15,8 \\
\hline Uranotaenia (Uranotaenia) geometrica & 0 & 0 & 4 & 4 & 0,3 \\
\hline Uranotaenia (Uranotaenia) palidoventer & 0 & 0 & 1 & 1 & 0,1 \\
\hline Uranotaenia (Uranotaenia) pulcherrima & 0 & 0 & 7 & 7 & 0,6 \\
\hline Wyeomyia (Dendromyia) luteoventralis & 1 & 0 & 4 & 5 & 0,4 \\
\hline Wyeomyia (Dendromyia) ypsipola & 0 & 0 & 1 & 1 & 0,1 \\
\hline \multirow[t]{2}{*}{ Wyeomyia autocratica } & 0 & 0 & 1 & 1 & 0,1 \\
\hline & 101 & 62 & 1.077 & 1.240 & 100,0 \\
\hline
\end{tabular}




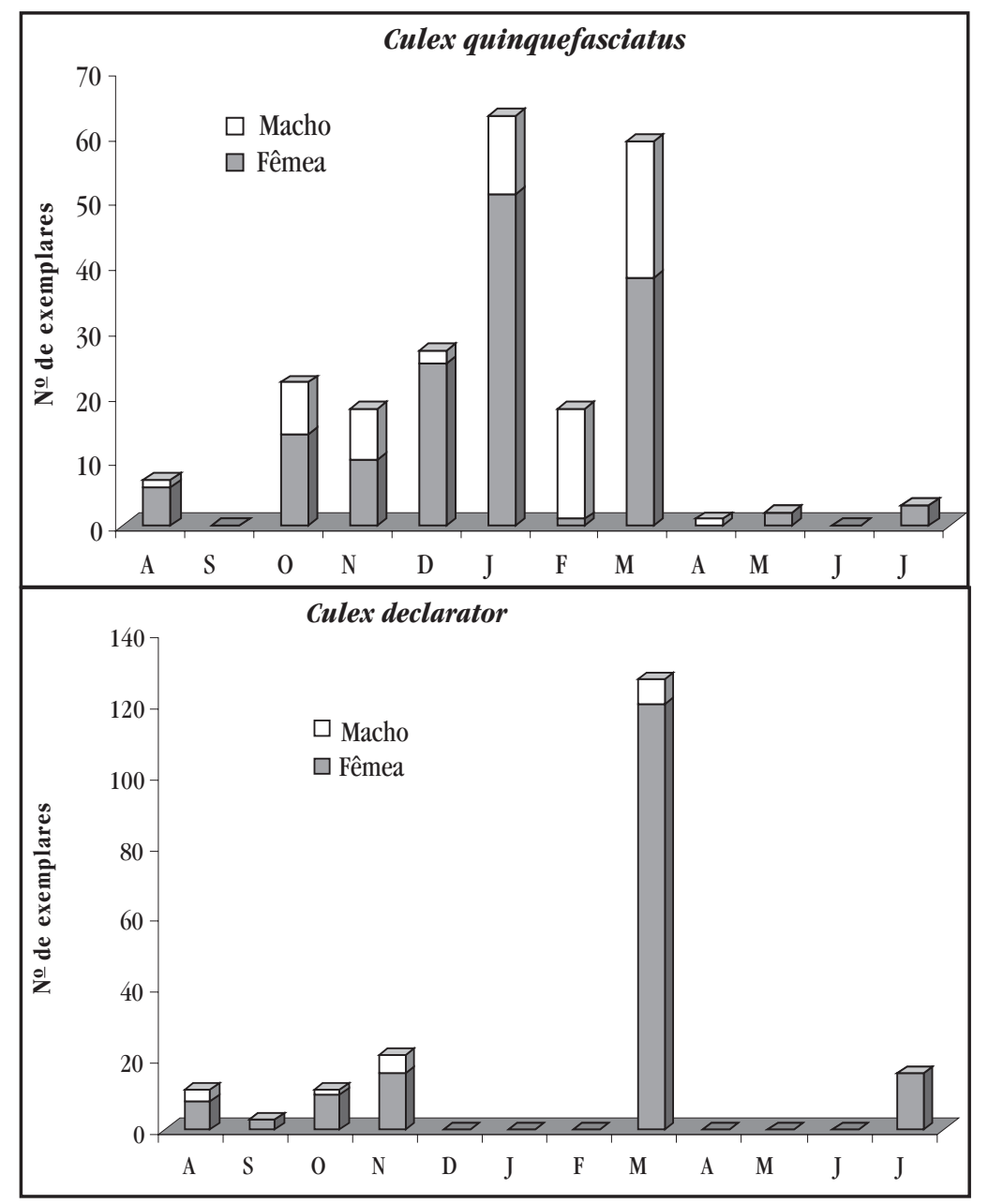

Figura 1 - Variação temporal da abundância das espécies de culicídeos incriminadas como vetoras mais freqüentes na Comunidade de São João, Manaus, Estado do Amazonas, de agosto de 2001 a julbo de 2002.

\section{DISCUSSÃO}

As espécies de culicídeos coletadas na Comunidade Rural São João já foram relatadas anteriormente no Estado do Amazonas $^{6133132}$. No presente estudo, foram coletadas 50 espécies de mosquitos, resultado semelhante ao encontrado em projeto de colonização recente na Bacia do Rio Purus, Estado do Acre ${ }^{20}$. Em municípios da zona rural do Estado do Amazonas, incluindo municípios vizinhos de Manaus, Fé e cols ${ }^{6}$, capturaram exemplares de 27 espécies. Hutchings e cols ${ }^{13}$, no Parque Nacional do Jaú, Estado do Amazonas, coletaram 130 táxons de culicídeos, o que aponta maior diversidade de espécies nos ambientes menos degradados.

Verificou-se 20 espécies do gênero Culex, que no conjunto somaram 904 (72,9\%) exemplares. A maior abundância e diversidade de espécies do gênero Culex está de acordo com estudo prévio no Estado do Amazonas ${ }^{13}$. Este gênero foi o que apresentou maior potencial de dispersão nos diferentes ecótopos de coletas. Apesar de predominar em ambiente florestal (1.104 exemplares; 85\%), também foi freqüente nos ambientes intradomiciliar e peridomiciliar (136; 15\%). Ressalta-se que 83,4\% dos culicídeos coletados nestes ecótopos pertencem ao gênero Culex. Apesar de Culex quinquefasciatus ter sido a espécie mais freqüente nos ambientes antrópicos, outras espécies deste gênero, em especial Culex declarator, parecem estar em via de domiciliação, representando possível elo entre o meio natural, os agentes que nele residem e a população humana.

A abundância de exemplares do gênero Culex, com representantes vetores de diversos agentes infecciosos, aponta o risco para infecção humana nas matas e mesmo no ambiente peridomiciliar da localidade. Culex usquatus foi registrada apenas no ambiente florestal, porém em elevada densidade. Embora até o presente momento não existam informações conclusivas sobre o envolvimento desta espécie na transmissão de agentes infecciosos, este achado sinaliza a necessidade de maiores estudos para o esclarecimento do real significado epidemiológico do seu registro, principalmente porque na região Amazônica foram isolados os vírus da encefalite de São Luís e o Mucambo ${ }^{34}$ e arbovírus causadores de doenças febris ${ }^{12}$ em Culex coronator, espécie do mesmo complexo de Culex usquatus.

Culex quinquefasciatus foi a segunda espécie mais coletada e mostrou capacidade de dispersão nos três ambientes de coletas, sendo a espécie predominante no intradomicílio, durante todos os meses do ano. Este achado confirma o que se 
conhece sobre o comportamento dessa espécie, que apresenta hábito acentuadamente domiciliado ${ }^{10}$. Em trabalhos anteriores, esta espécie também predominou nas coletas realizadas no intradomicilio ${ }^{21} 27$. Sua presença é motivo de incômodo e risco para a população humana por ser o mais eficiente vetor da filariose bancroftiana ao homem ${ }^{10}$ e por ter sido encontrada naturalmente infectada pelo vírus da encefalite de São Luís ${ }^{19}$ e pelo vírus Oropouche ${ }^{23}$. A presença de elevadas densidades deste mosquito possivelmente se deve à ausência de rede de abastecimento e destinos de esgotos e da água de descarte doméstico na comunidade, promovendo a formação de criadouros peridomiciliares. Adicionalmente, os recipientes artificiais eliminados para o ambiente podem ser utilizados como hábitat larvário, propiciando sua adaptação ao ambiente antrópico ${ }^{15} 16$

Deve-se mencionar, ainda, o encontro das espécies Culex nigripalpus e Culex declarator nos ambientes domiciliares. A primeira espécie foi incriminada como vetor do vírus da encefalite de São Luís na América Central e norte da América do $\mathrm{Sul}^{22}$ e suspeita-se que a segunda possa intervir no ciclo natural desse mesmo agente na Amazônia Brasileira ${ }^{34}$. Note-se que de acordo com Labarthe e cols ${ }^{14}$, há possibilidade de Culex declarator ser vetor de Dirofilaria immitis.

No gênero Psorophora destacou-se Psorophora amazonica, principalmente no ambiente florestal. Possivelmente este gênero contribua na disseminação de arbovírus em condições naturais, a exemplo dos vírus Ilhéus, Mayaro, encefalite equiina Oeste, Rocio, encefalite eqüina Venezuelana e outros ${ }^{33}$.

No que se refere às espécies Anopheles darlingi e Anopheles triannulatus, foi constatado baixa densidade, provavelmente pelos métodos de coleta empregados, que não incluíram o emprego de isca humana. Vale ressaltar que no ano de 2001 foi registrado o menor número de notificações de casos de malária dentre os últimos 19 anos $^{28}$, possivelmente pela intensificação das ações de combate ao vetor na Amazônia Legal entre 1999 e $2000^{18}$, podendo ter refletido na diminuição da densidade populacional de anofelinos dessa área. Anopheles darlingi, principal transmissora de malária no Brasil, é uma espécie de hábito antropofílico e a sua densidade populacional é menor nas áreas nativas do que nas áreas invadidas pelo homem ${ }^{2632}$. Na área investigada registrou-se a presença de Haemagogus janthinomys e outras espécies de Sabethini que podem manter o ciclo enzoótico natural da febre amarela silvestre, uma vez que atuam na veiculação do vírus amarílico $^{310}$. Nesse contexto, a proximidade das habitações daquela comunidade com a floresta primária, onde se registrou a presença de Haemagogus janthinomys, aumenta o risco da incidência da doença na população, uma vez que naquela área muitos moradores freqüentam as matas com o hábito de extração de madeira e caça. De 1980 a 2005, foram registrados 53 casos de febre amarela no Estado do Amazonas, com número crescente na última década e alta taxa de mortalidade, o que representa $7,9 \%$ dos casos no Brasil e $21 \%$ dos casos da região norte, no mesmo período ${ }^{17}$.

0 registro de Aedes aegypti nas formas imaturas e adultas, caracteriza a possibilidade de dispersão desta espécie para comunidades rurais. Nestas localidades observam-se condições para o estabelecimento desta espécie, mesmo que temporariamente, por meio da disponibilidade de potenciais criadouros artificiais, pela inexistência de serviço de coleta de lixo. Este fato pode ter importante repercussão epidemiológica, pois o intenso fluxo populacional entre a zona urbana de Manaus com os aglomerados populacionais da zona rural do município possibilita a transposição antrópica dessa espécie para estas áreas, tornandoas potenciais focos de dengue e febre amarela. 0 registro de Haemagogus janthinomys nas áreas de matas localizadas a menos de $100 \mathrm{~m}$ das residências e encontro de exemplares de Aedes aegypti no intradomicílio evidencia a vulnerabilidade dessa população à febre amarela. 0 homem, ao entrar na mata para o exercício do extrativismo, pode eventualmente infectar-se do vírus silvestre e contribuir com sua disseminação, inclusive para ambientes urbanos, como ocorreu na cidade boliviana de Santa Cruz de la Sierra ${ }^{29}$.

Apesar da baixa densidade de culicídeos, foi registrada a presença de diversas espécies incriminadas como vetoras de agentes etiológicos, inclusive no ambiente domiciliar. Este fato requer atenção especial por parte dos órgãos de saúde e aponta para a necessidade de estudos adicionais abordando aspectos bioecológicos para maior conhecimento das relações dessas espécies com seus habitats e avaliação do seu potencial de disseminação para os ambientes domiciliares.

Os estudos da fauna e comportamento de culicídeos são de grande relevância epidemiológica, pois podem fornecer dados que irão subsidiar as ações de combate e controle de espécies vetoras de agentes patogênicos, pelos órgãos competentes. A riqueza de espécies envolvidas na transmissão de doenças endêmicas e emergentes nessa área revela a necessidade de uma vigilância entomológica permanente.

\section{AGRADECIMENTOS}

Aos técnicos de entomologia Flávio Augusto Andrade Fé, Orleans Furtado Pastor e ao motorista Elcimar Neves Cavalcante, pelo auxílio nas coletas de campo. Aos moradores da Comunidade São João, particularmente ao Mauro Silva, pelo auxílio durante a execução do Projeto.

\section{REFERÊNCIAS}

1. Barbosa OC, Teodoro U, Lozovei AL, La Salvia Filho V, Spinosa RP, Lima EM, Ferreira MEMC. Nota sobre culicídeos adultos coletados na região sul do Brasil. Revista de Saúde Pública 27:214-216, 1993.

2. Cerqueira NL. Cinco Novos Sabetinos da Amazônia (Diptera), Culicidae). Revista Brasileira de Entomologia 10:37-52, 1961.

3. Consoli RAGB, Lourenço-de-Oliveira RL. Principais Mosquitos de Importância Sanitária no Brasil. Fundação Instituto Oswaldo Cruz, Rio de Janeiro, 1994.

4. Deane LM. Malaria vectors in Brazil. Memórias do Instituto Oswaldo Cruz 81:5-14, 1986

5. Faran ME. A revision of the Albimanus Section of the genus Nyssorhynchus of Anopheles. Mosquito Studies XXXIV. American Entomological Institute 15:1-215, 1980. 
6. Fé NF, Barbosa MGV, Fé FAA, Guerra MVF, Alecrim WD. Fauna de Culicidae em municípios da zona rural do Estado do Amazonas, com incidência de febre amarela. Revista da Sociedade Brasileira de Medicina Tropical 36:343-348, 2003 .

7. Forattini OP. Entomologia Médica. Volume 1. Editora da Universidade de São Paulo, São Paulo, 1962.

8. Forattini OP. Entomologia Médica. Volume 2. Editora da Universidade de São Paulo, São Paulo, 1965.

9. Forattini OP. Entomologia Médica. Volume 4. Editora da Universidade de São Paulo, São Paulo, 1965.

10. Forattini OP. Culicidologia Médica. Editora da Universidade de São Paulo, São Paulo, 2003.

11. Forattini OP, Kakitani I, Massad D, Studies on mosquitoes (Diptera: Culicidae) and anthropic environment. 2. Immature stages research at a rice irrigation system location in South-Eastern Brazil. Revista de Saúde Pública 27:227-236, 1993.

12. Hervé JP, Dégallier N., Travassos-da-Rosa APA, Pinheiro FP, Sá-Filho GC. Arboviroses - Aspectos ecológicos. In: Instituto Evandro Chagas - 50 anos de contribuição às ciências biológicas e à medicina tropical. Fundação do Serviço de Saúde Pública, Belém, 1986.

13. Hutchings RSG, Sallum MAM, Ferreira RLM, Hutchings RW. Mosquitoes of the Jaú National Park and their potential importance in Brazilian Amazonia Medical and Veterinary Entomology 19:428-441, 2005.

14. Labarthe N, Serrão ML, Melo FY, de Oliveira SJ, Lourenço-de-Oliveira R. Potencial vectors of Dirofilaria immitis (Leidy, 1856) in Itacoatiara, Oceanic Region of Niterói municipality, State of Rio de Janeiro, Brazil. Memórias do Instituto Oswaldo Cruz 93:425-432, 1998.

15. Lopes J. Ecologia de mosquitos (Diptera: Culicidae) que procriam em criadouros naturais e artificiais em área rural no Norte do Paraná, Brasil. V. Coleta de larvas em recipientes artificiais instalados na mata ciliar. Revista de Saúde Pública 31:370-377, 1997.

16. Lopes J. Ecologia de mosquitos (Diptera: Culicidae) que procriam em criadouros naturais e artificiais em área rural no Norte do Paraná, Brasil. VII. Coexistência das espécies. Iheringia - Série Zoologia 83:91-97, 1997.

17. Ministério da Saúde. Febre Amarela - Distribuição de casos confirmados, por Unidade Federada, Brasil, de 1980 a 2005. http://dtr2001.saude.gov.br/svsepi/ situacao_doencas. Acessado em 01/04//2008, 2008.

18. Ministério da Saúde. Plano de intensificação das Ações de Controle da Malária na Amazônia Legal. Relatório Executivo. Ministério da Saúde, Brasília, 2002.

19. Mitchell CJ, Monath TP, Sabattini MS, Cropp CB, Daffner JF, Clisher CH, Jakob WL, Christenses HÁ. Arbovirus investigations in Argentina, 1977-1980. II. Arthropod collections and vírus isolations from argentine mosquitoes. American Journal of Tropical Hygiene 34:945-55, 1985.

20. Natal D, Barata JMS, Taipe-Lagos CB, Rocha RM. Nota sobre culicídeos (Diptera: Culicidae) da bacia do rio Purus, Acre, Amazônia (Brasil). Revista de Saúde Pública 26:129-131, 1992.
21. Naves HAM, Carvalho MESD, Costa JA, Oliveira RA. Frequiência domiciliar de Culicidae em zona urbana de Goiânia-Goiás-Brasil. Revista de Patologia Tropical 25:43-9, 1996.

22. Nayar JK. Bionomics and physiology of Culex quinquefasciatus (Diptera:Culicidae) of Florida: An important vector of disease. University of Florida, Gainesville, 1982.

23. Pinheiro FP, Travassos-da-Rosa APA, Vasconcelos PFC. An overview of Oropouche fever epidemics in Brazil and neighbour countries. In: Travassos-da-Rosa APA, Vasconcelos PFC, Rosa JFST (eds). An overview of arbovirology in Brazil and neighbouring countries. Instituto Evandro Chagas, Belém, p. 186-192, 1998.

24. Queiroz CAB, Tadei WP, Terrazas W, Baggio JB, Nunes-Filho HP, Lopes NR, Oliveira AEN, Figueiredo EO. Malária em Manaus: epidemias e suas relações com a expansão desordenada de ocupação periurbana. Revista da Sociedade Brasileira de Medicina Tropical 34 (supl. 1):73, 2001.

25. Rachou RG. Anofelinos do Brasil: Comportamento das espécies vetoras da Malária. Revista Brasileira de Malariologia e Doenças Tropicais 10:145-181, 1958.

26. Rebelo JMM, Silva AR, Ferreira LA, Vieira JA.Anopheles (Culicidae, Anophelinae) e a malária em Buriticupu - Santa Luzia, Pré-Amazônia maranhense. Revista da Sociedade Brasileira de Medicina Tropical 30(2):107-111, 1997.

27. Reisen WK, Milby MM, Meyer RP. Population dynamics of adult Culex mosquitoes (Diptera: Culicidae) along the Kern River, Kern County, California, in 1990. Journal of Medical Entomology 29:531-543, 1992.

28. Saraiva MGG. Malária em Manaus: Análise epidemiológica, conhecimento e atitudes da população. Dissertação de Mestrado, Universidade do Estado do Amazonas, Manaus, AM, 2007.

29. Stuyft PV, Gianella A, Pirard M, Cespedes J, Lora J, Peredo C, Pelegrino JL, Vorndam V, Boelaert M. Urbanisation of yellow fever in Santa Cruz, Bolivia. Lancet 353:1558-1562, 1999 .

30. Tadei WP, Mascarenhas BM, Podestá MG. Biologia de anofelinos amazônicos. VIII. Conhecimentos sobre a distribuição de espécies de Anopheles na região de Tucuruí-Marabá (Pará). Acta Amazônica 13:103-140, 1983.

31. Tadei WP, Thatcher BD. Malaria vectors in the Brazilian amazon: Anopheles of the subgenus Nyssorhynchus. Revista do Instituto de Medicina Tropical de São Paulo 42: 87-94, 2000.

32. Tadei WP, Thatcher BD, Santos JM, Scarpassa VM, Rodrigues IB, Rafael MS. Ecologic observations on anopheline vectors of malaria in the Brazilian Amazon. The American Journal of Tropical Medicine and Hygiene 59:325-335, 1998.

33. Travassos-da-Rosa ES. Arboviruses pathogenic for man in Brazil In: Travassos APA, Vasconcelos PFC, Rosa JFST (eds) An overview of arbovirology in Brazil and neighboring countries. Instituto Evandro Chagas, Belém, p.72-99, 1998.

34. Vasconcelos PFC, Rosa JT, Travassos-da-Rosa APA, Dégallier N, Pinheiro FP, Sá-Filho GC. Epidemiologia das encefalites por arbovírus na Amazônia brasileira. Revista do Instituto de Medicina Tropical de São Paulo 33:465-476, 1991.

35. Zequi JAC, Lopes J, Medri ÍM. Imaturos de Culicidae (Diptera) encontrados em recipientes instalados em mata residual no munícipio de Londrina, Paraná, Brasil. Revista Brasileira de Zoologia 22:656-661, 2005. 